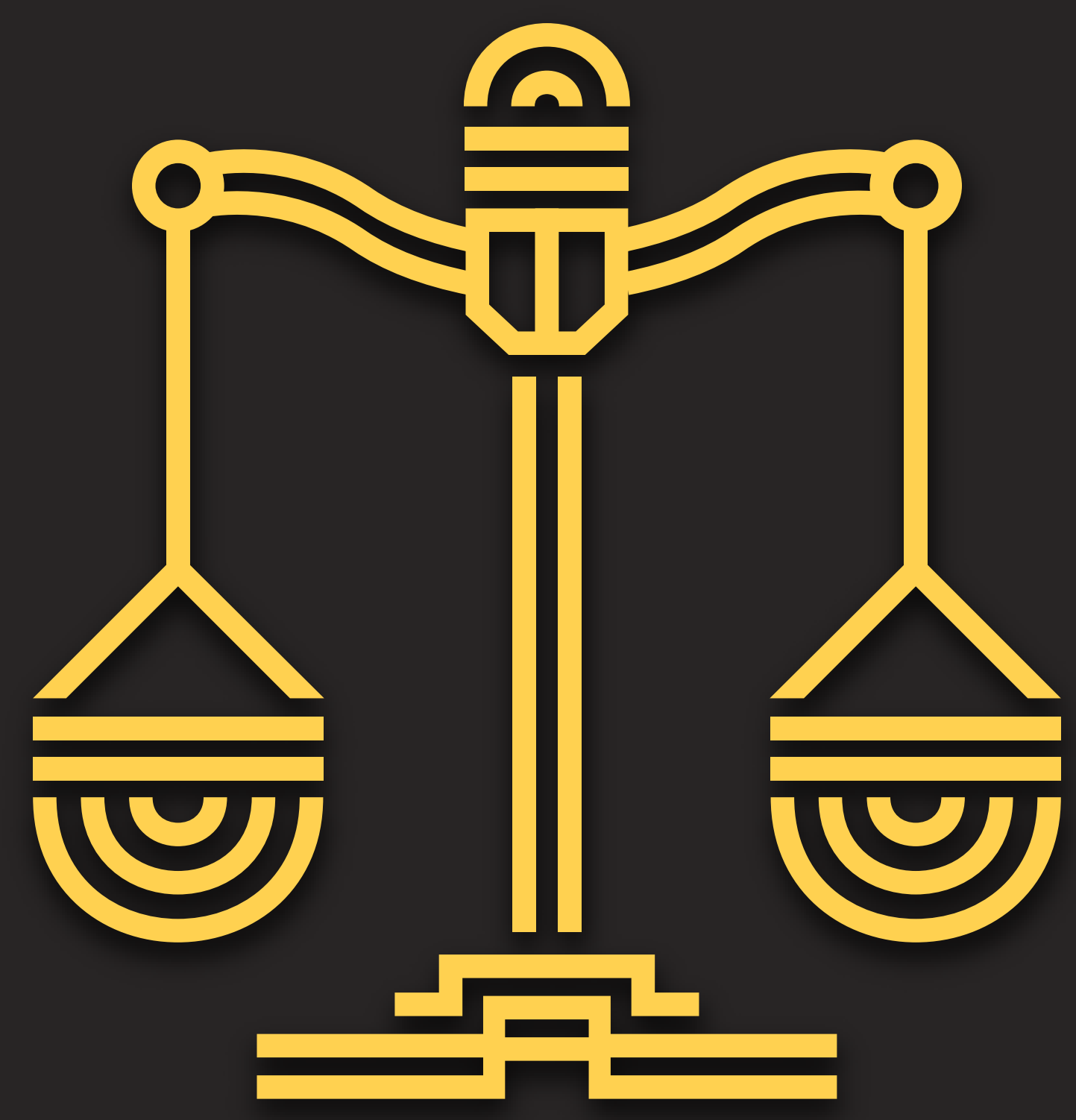

\title{
BOLETÍN INFORMATIVO
}

\section{"IURE TEMPUS I. Facultad de Derecho}

EDICIÓN N. ${ }^{\circ} 1$ 


\section{COMITÉ EDITORIAL}

Fray Carlos Arturo díAz RODRíGUEZ, O. P.

Decano de la División de Ciencias Jurídicas y Políticas

Doctor Ciro Nolberto Güechá Medina

Decano Académico de la Facultad de Derecho

Doctor Julián Alberto Becerra García

Secretario de la División de Ciencias Jurídicas y Políticas

Doctora Norhys Esther Torregrosa Jiménez

Directora del Centro de Investigación de la Facultad de Derecho

Doctora Dalia Carreño Dueñas

Directora del semillero de investigación

Hermenéutica y Argumentación Jurídica

Daniela Alejandra Valencia González

Monitora del Centro de Investigación

Esteban José Ahumada Quiroga

Monitor del Centro de Investigación 


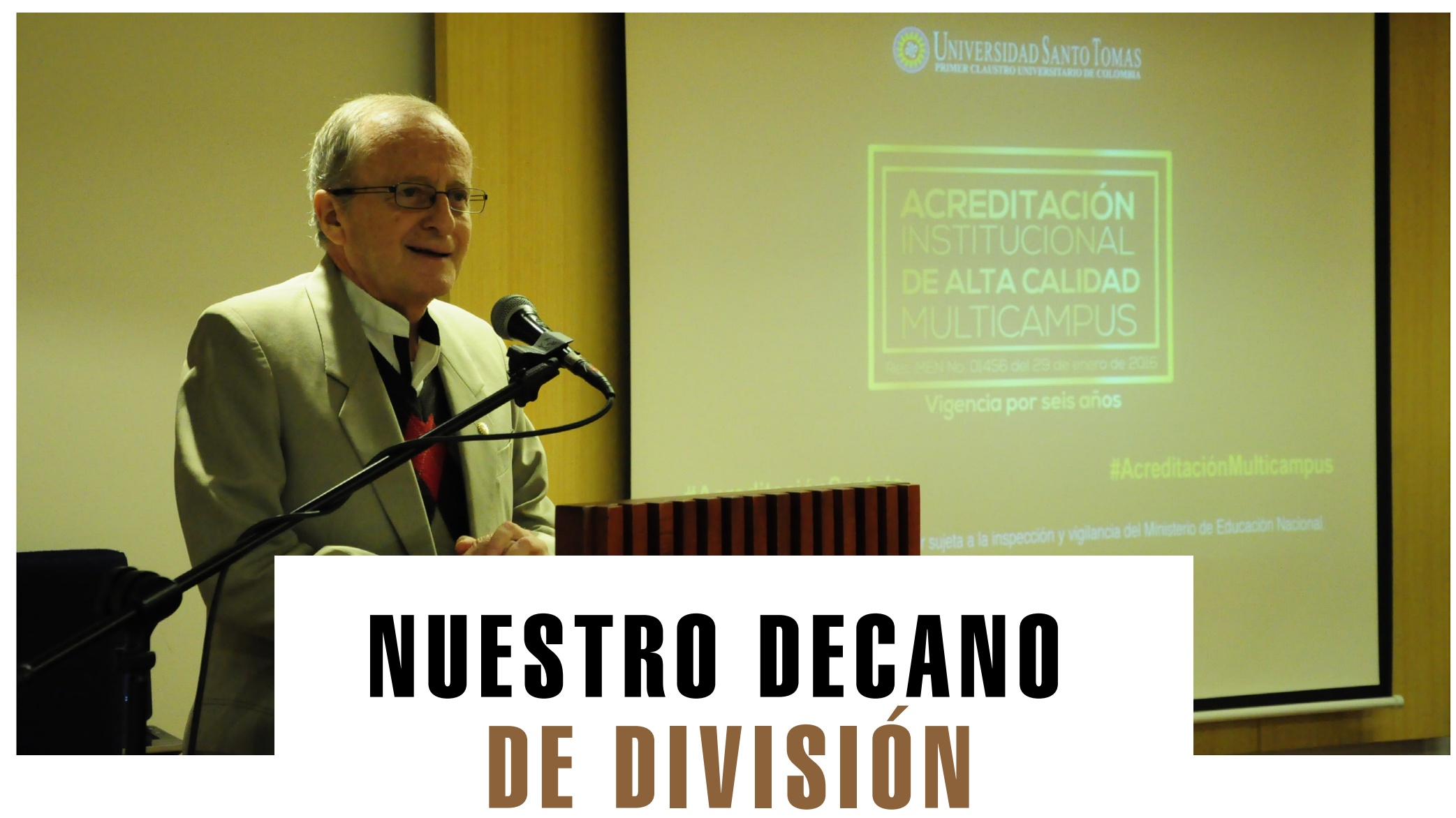

Fray Carlos Arturo díAZ RODRíGUEZ, O. P., actual Decano de la División de Ciencias Jurídicas y Políticas, es Ingeniero Químico de la Universidad Nacional de Colombia. Estudió Filosofía en la Universidad Santo Tomás y Teología en la Pontificia Universidad Javeriana. Es Licenciado en Derecho Canónico del Angelicum en Roma; Magíster en Administración de Empresas de la Universidad Santo Tomás; Investment de la Universidad Marquette; Doctor en Administración de Negocios de la Universidad de New York; y Doctor en Economía y Administración de Empresas de la Universidad Nacional de Educación a Distancia (UNED) de España.

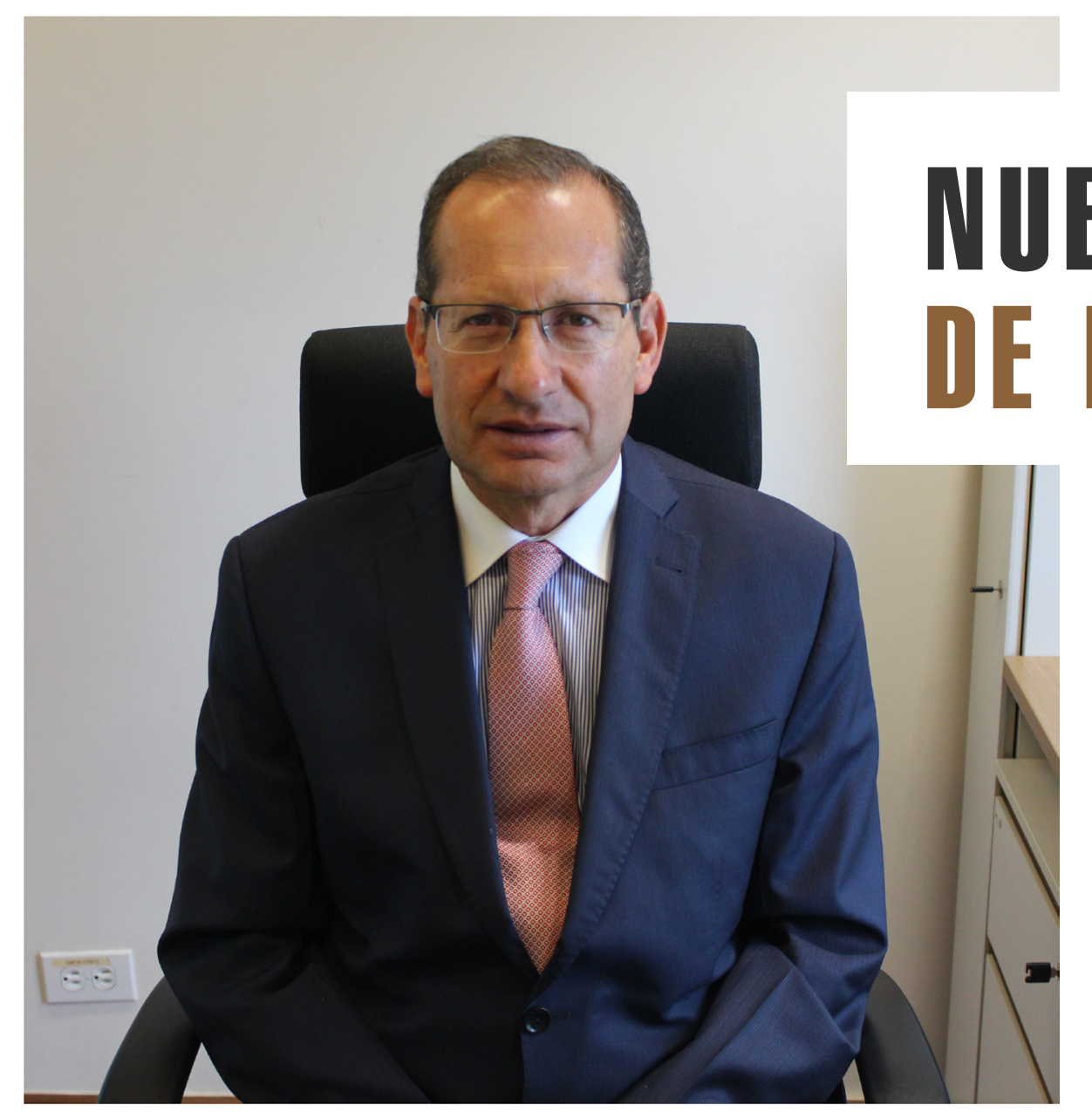

Ciro Nolberto Güechá Medina es Doctor en Derecho de la Universidad Externado de Colombia y Doctor en Derecho Administrativo de la Universidad Alfonso X el Sabio de España. Cuenta con numerosas publicaciones en revistas indexadas y libros científicos. 


\section{DESTAGADOS DEL PROGRAMA}

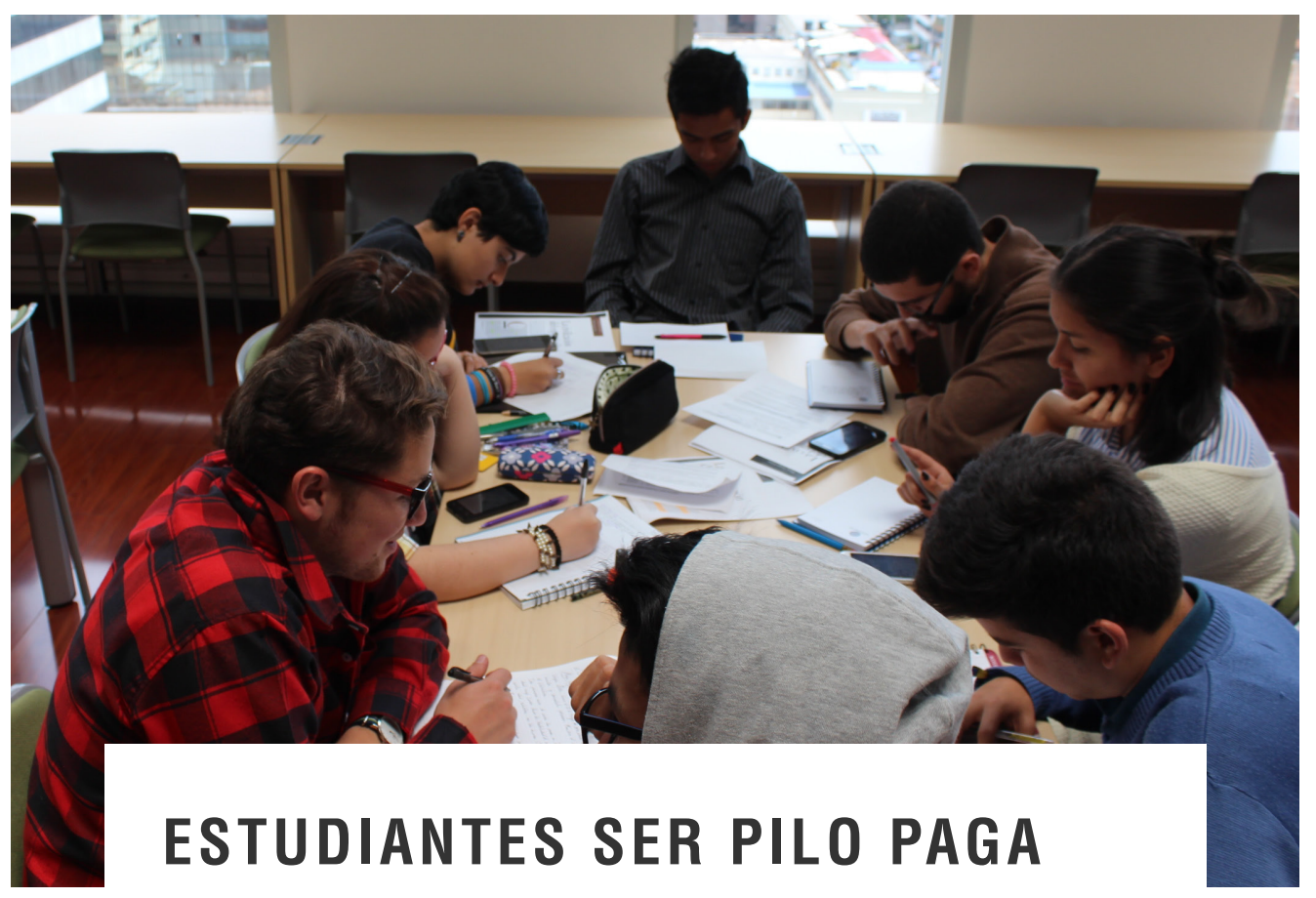

Desde el año 2015 la Facultad de Derecho se unió a la campaña de implementación educativa de nuevas oportunidades, a través del programa Ser Pilo Paga, abriendo las puertas al cambio y a la inclusión, permitiendo así que se enriquezca la comunidad tomasina.

Si bien la campaña se inició con pocos cupos, se desarrolló de manera satisfactoria, iniciando con 17 estudiantes en el primer año, ascendiendo a 59 en el segundo periodo y culminando en la actualidad con un total aproximado de 76 estudiantes que han podido acceder a la Educación Superior en la Universidad Santo Tomás.
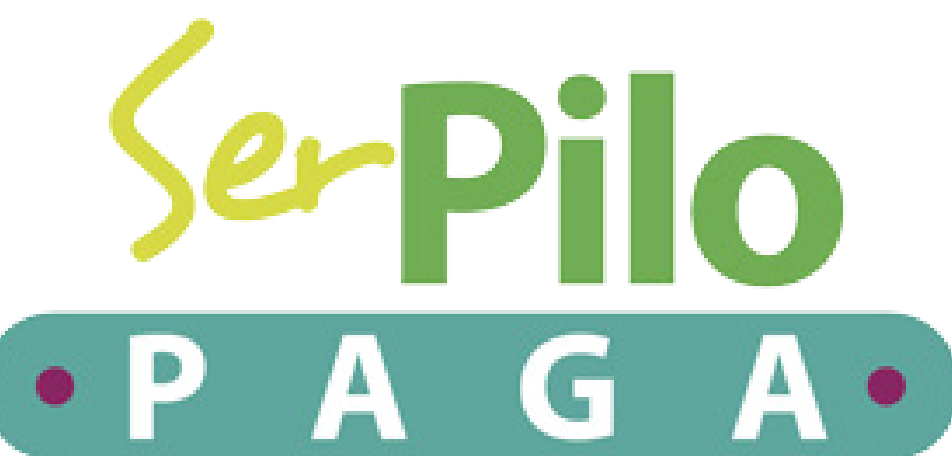

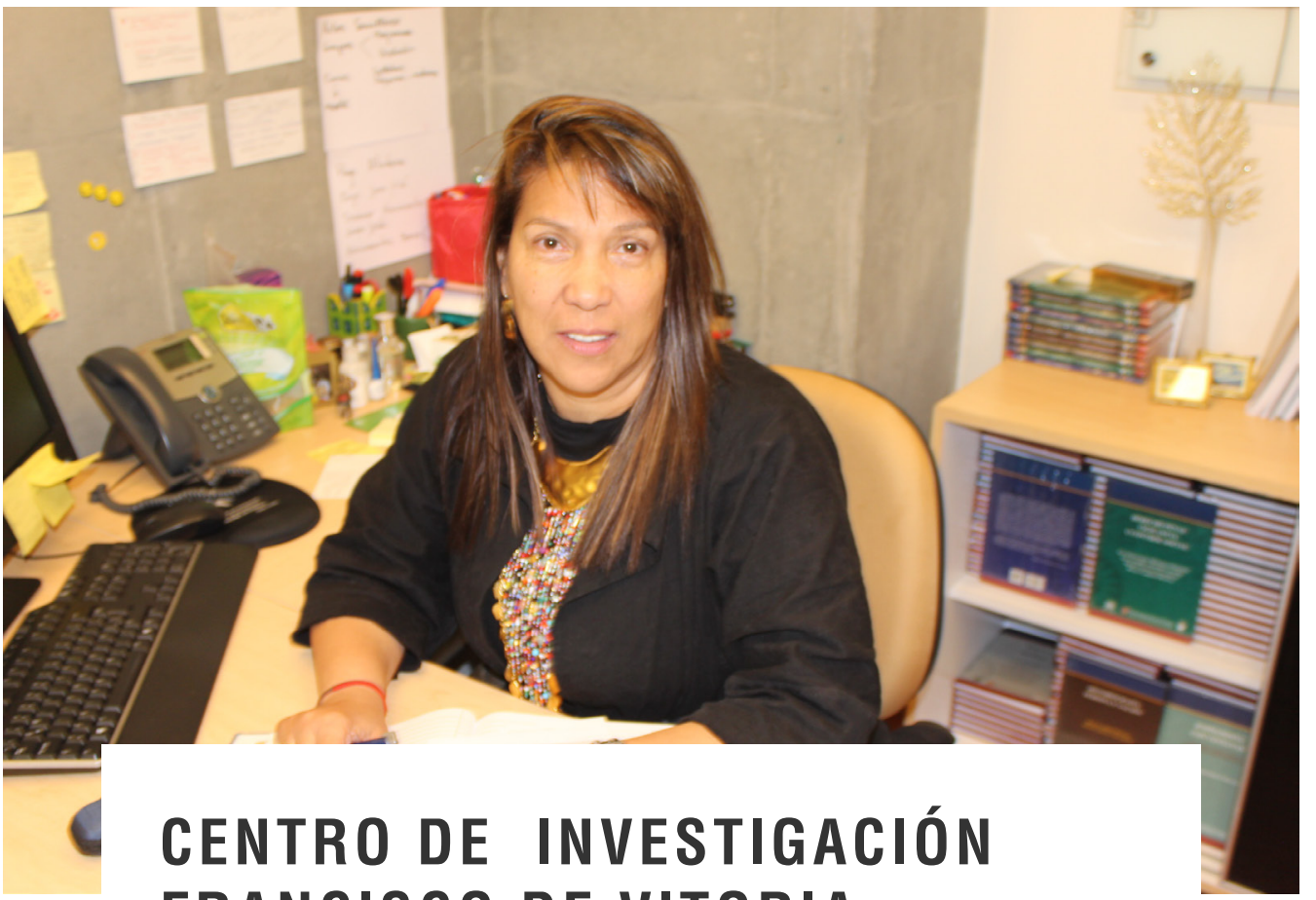
FRANCISCO DE VITORIA

El centro de Investigación Francisco de Vitoria es un espacio que fomenta la investigación con la participación de cuatro grupos de categorizados en Colciencias y nueve semilleros.

Te invitamos a que hagas parte de esta familia de investigadores inscribiéndote enlas diferentes actividades que propone el centro.

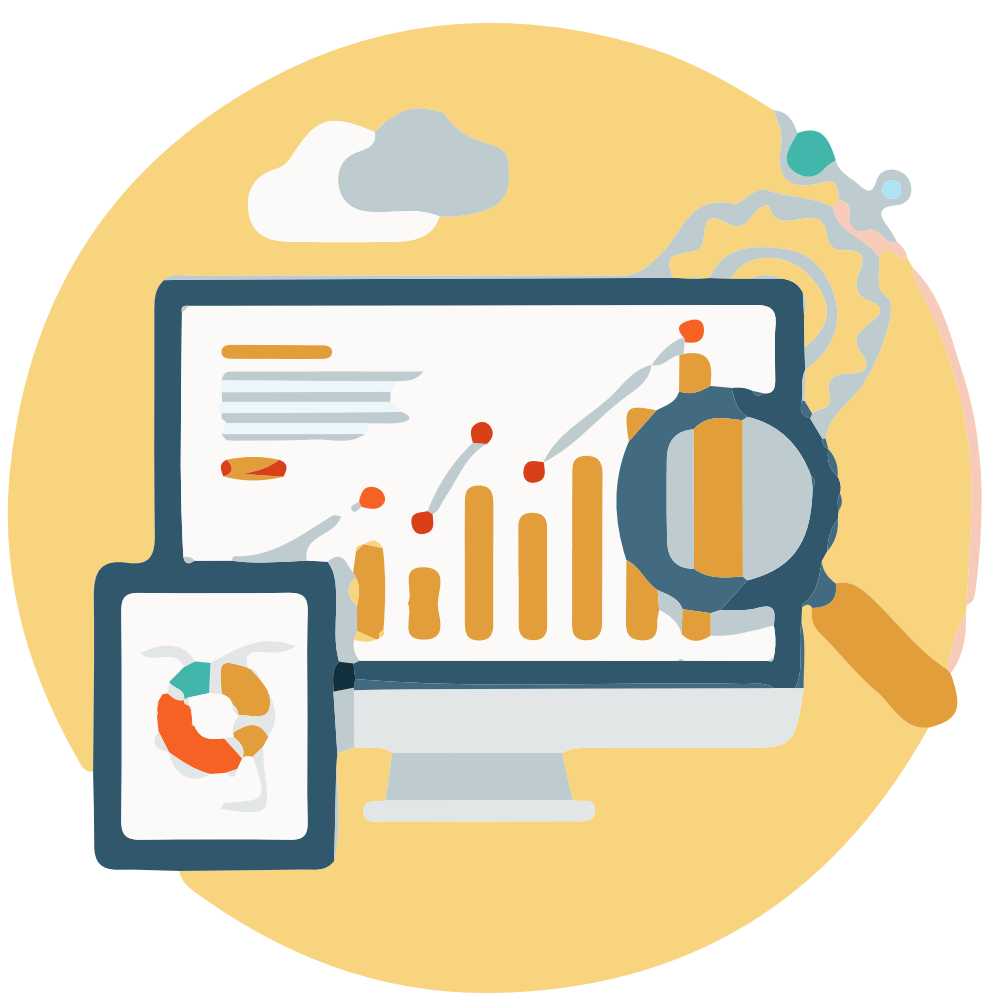




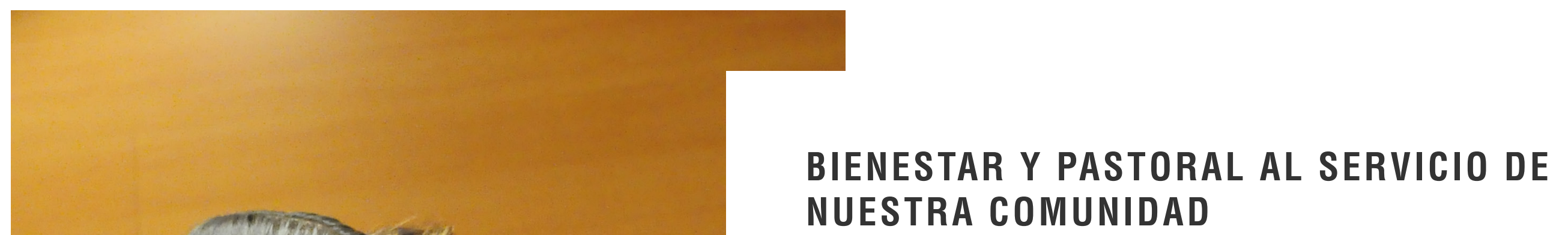

Dentro de la Universidad Santo Tomás existe una dependencia encargada del cuidado y la atención de la comunidad universitaria durante su paso por la Institución, se trata del Departamento de Promoción y Bienestar Universitario.

De la mano de Rosemary Ramos Oliveros y Laura Cuellar, en las instalaciones del Edificio Doctor Angélico, los tomasinos pueden desarrollar actividades culturales, deportivas y de dispersión como una vía para salir de la rutina.

Por otra parte, con el Centro de Pastoral Universitaria la comunidad tiene un espacio para conectarse con la realidad social por la que actualmente atraviesa el país, Iogrando intervenir y favorecer con actividades que le apuntan al cambio.

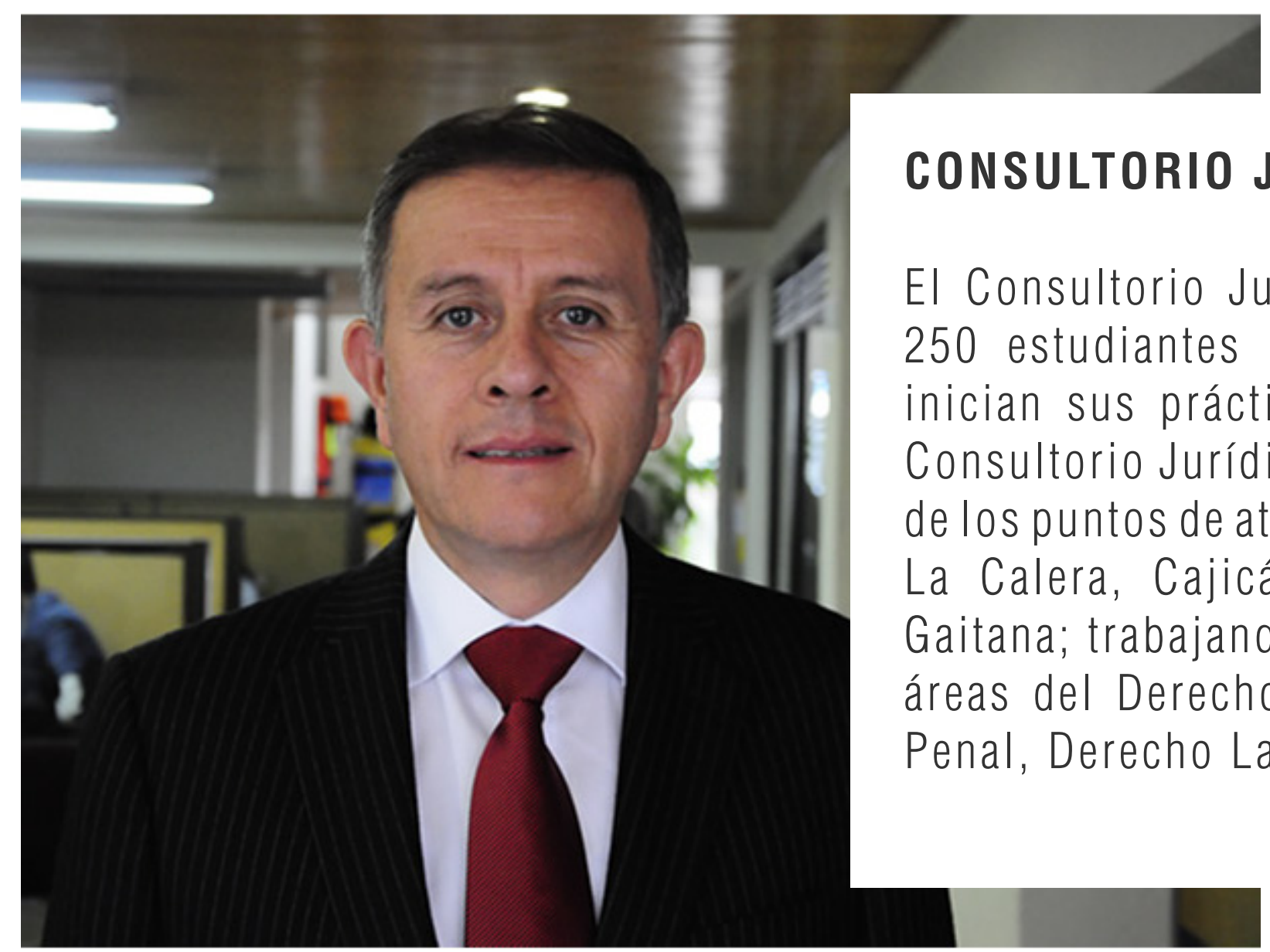




\section{NOTIGIAS DE LA FAGULTAD}

\section{HUMANISMO, DERECHO Y POSCONFLICTO \\ EN LA BIENVENIDA A NEOTOMASINOS DE LA FACULTAD DE DERECHO}

El día 8 de agosto, dentro del marco del foro 'Humanismo, Derecho y Posconflicto', se realizó la bienvenida a los neotomasinos de la Facultad de Derecho, un evento que contó con la participación de todos sus estudiantes.

El acto inició con el saludo enviado por fray Carlos Arturo DÍAZ RODRÍGUEZ, 0. P., Decano de la División de Ciencias Jurídicas y Políticas, quien se refirió al orgullo que significa ser tomasino de corazón y que conlleva sin duda a que cada futuro egresado lleve en alto los valores humanistas, con lo cual han de imprimir la impronta de un nuevo país con justicia y paz social.

Luego, el Decano de la Facultad de Derecho, Dr. Ciro Güechá Medina, señaló que uno de los propósitos de la formación de la facultad es que sus egresados sean excelentes como personas y como juristas; que asuman el reto no solo de ser abogados, pues otras universidades lo pueden hacer, sino de ser auténticos juristas, es decir, creadores, pensadores de un derecho humanista, para una sociedad más justa, inclusiva y participativa, que aporte más allá del posconflicto.

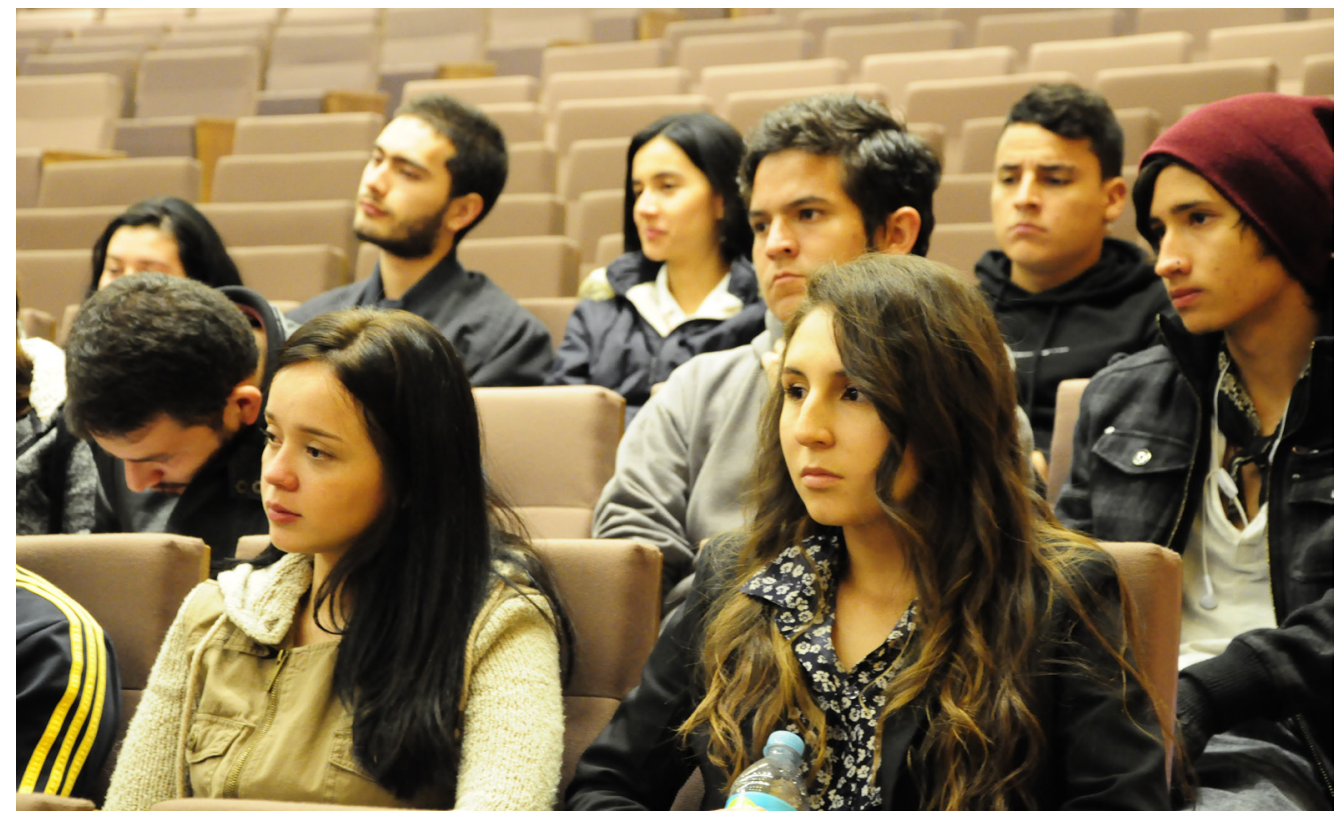

Un jurista que logre atender las verdaderas necesidades del acceso a la justicia como contribución al Estado Social de Derecho, y que como persona asuma el compromiso por ser cada día un ser humano virtuoso y disciplinado.

También invitó a que los neotomasinos de la USTA sobresalgan por sus actitudes éticas y creativas como lo dicta la misión institucional, con la cuales se contribuya a la construcción de la sociedad del posconflicto.

Mientras tanto, la Coordinadora del Módulo, Dalia Carreño Dueñas, señaló el rol protagónico que tienen los neotomasinos en la instauración de la nación del posconflicto y para ello han de asumir con responsabilidad el ser los mejores juristas del país, para lo cual se requiere que sean personas honestas, justas y ante todo cumplidoras de la norma, pues solo quien obedece las leyes puede gobernar; invitó a los estudiantes a dar lo mejor de sí, y a llevar con honor y dignidad el ser tomistas.

El Dr. Edgar Guarín fue el encargado de profundizar en el sistema modular como el sello característico de la USTA, pues se trata de un 
currículo propio que desde el año 1975 se ha venido actualizando y perfeccionado; el método que guía esta forma de comprender, asumir el derecho y su forma de enseñanza es el ver, juzgar y actuar, que se expresa en los pasos que se han de trazar para los fines del sistema modular, y que inspirados en el realismo de Tomás de Aquino se configuran como materia modular, centro de interés y eje temático.

Por lo que se ve, la materia modular se juzga a la luz de los valores en juego desde el centro de interés y se actúa desde el eje temático, configurándose en una verdadera opción para leer, juzgar y actuar en el contexto del posconflicto.

La Dra. Norhrys Torregrosa, Directora del Centro de Investigaciones Francisco Vitoria (CIFRAVI), centró su intervención en que los nuevos perfiles del jurista tienen que ver con la necesidad de investigar, entre otros, el objeto material del derecho, es decir, la realidad, y con ello contribuir con las exigencias de repensar los valores fundantes del orden legal que demanda un país que hace apuestas por el posconflicto. Presentó a los neotomasinos el elemento transversal de la investigación que inicia en primer semestre y culmina en octavo con la entrega del trabajo de investigación que le permitirá cumplir uno de los requisitos de grado como abogado de la Universidad Santo Tomás.

El estudiante Daniel Alvarado, como Representante de los Estudiantes, presentó su saludo de bienvenida a los neotomasinos señalando aspectos generales del Reglamento Estudiantil y la necesidad de asumir el compromiso de ser tomasino de corazón. El evento cerró con la intervención de Rosemary Ramos, Sandra Guillén y Vilma Moreno, del Departamento de Promoción y Bienestar Universitario, Ia UDIES y la Consejería Estudiantil de la Facultad, todas como equipo de

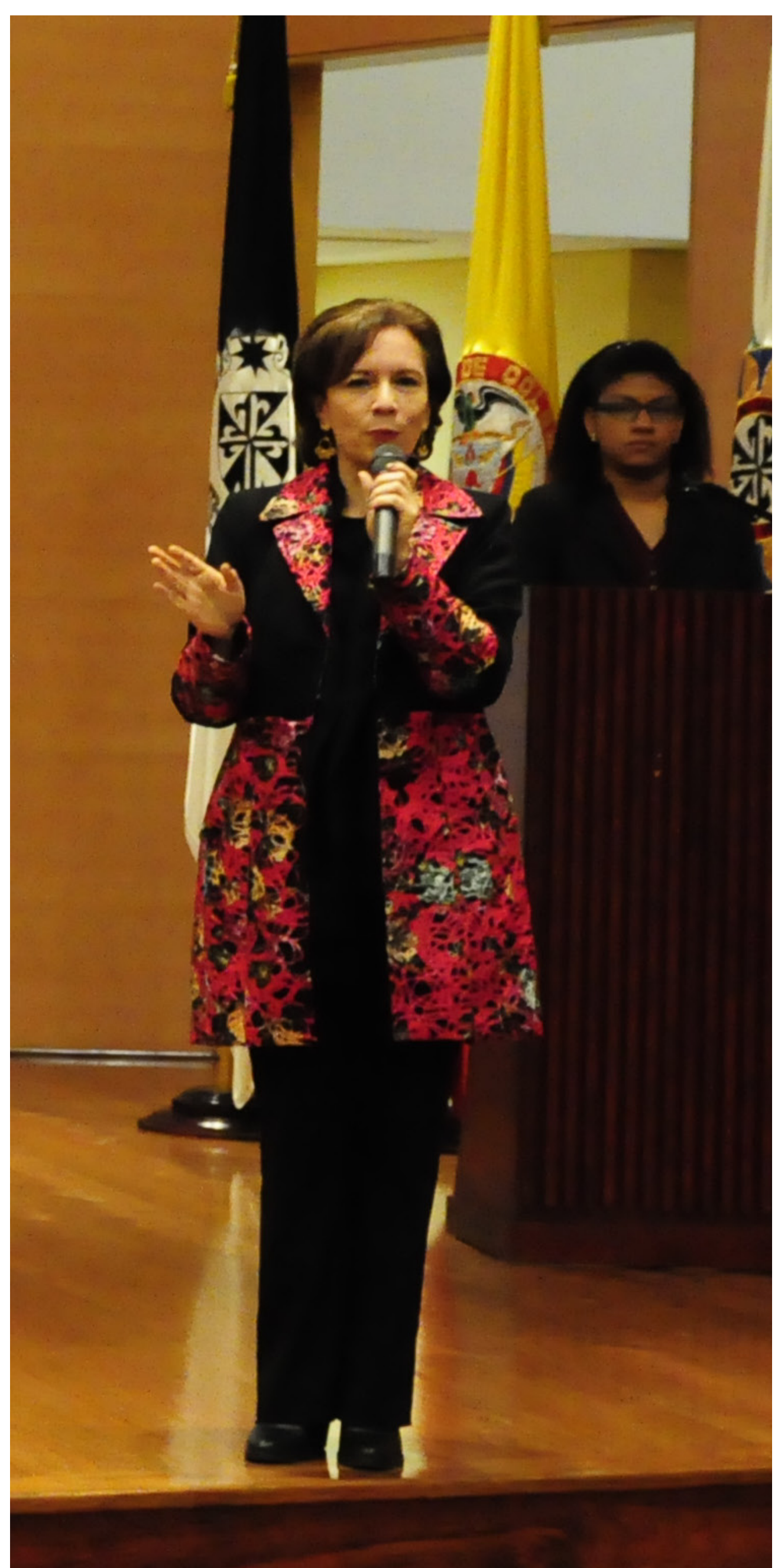

acompañamiento y colaboración con el proyecto de excelencia del neotomasino.

Noticia publicada en Tomás Noticias - 30 de agosto de 2017 


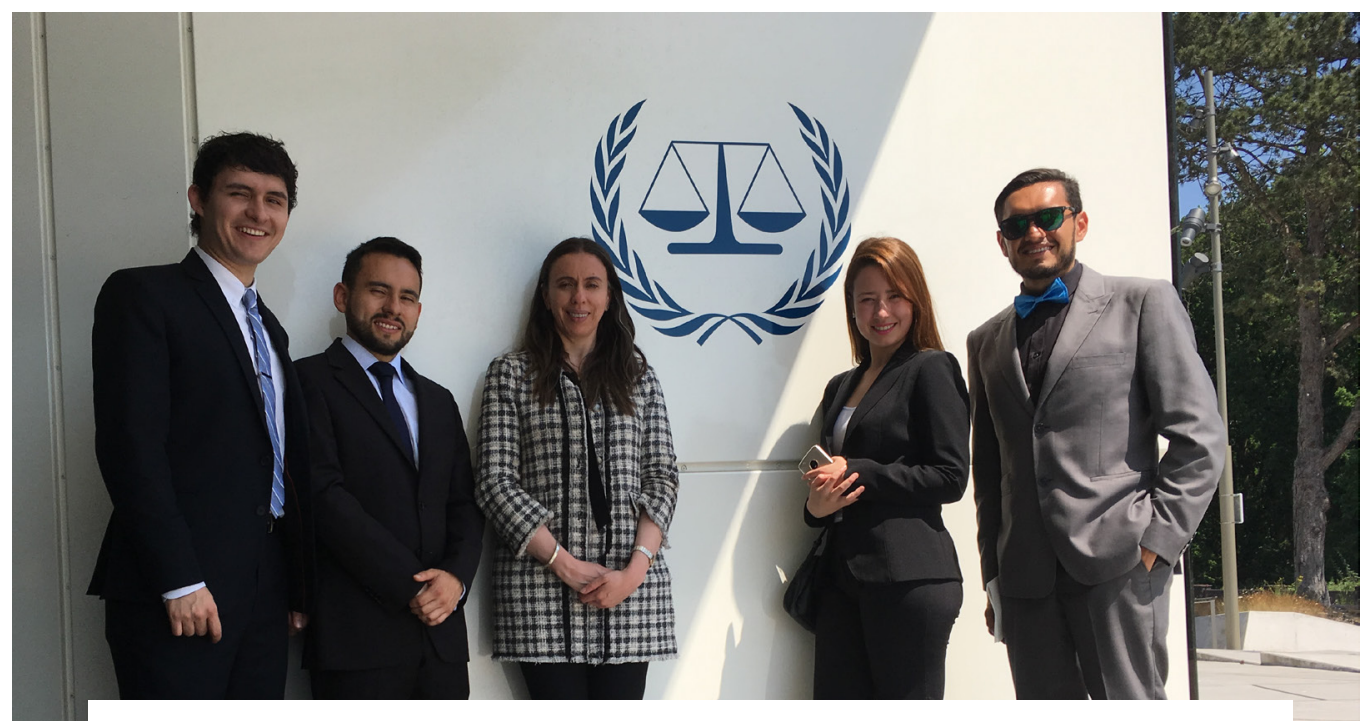

\section{PARTICIPACIÓN EN LA V EDICIÓN DEL CONCURSO DE SIMULACIÓN JUDICIAL ANTE LA CORTE PENAL INTERNACIONAL}

Daniel Alvarado Lugo, María José Hernández Mejía y César Gaitán Peñalosa son los estudiantes de la Facultad de Derecho que el pasado mes de junio representaron a la Universidad en la V Edición del Concurso de Simulación Judicial ante la Corte Penal Internacional, que se realizó en La Haya, Holanda.

Gracias al entrenamiento de la Dra. Sandra Patricia Ramírez Montes, docente e investigadora líder del semillero de Jurisprudencia Penal Internacional de dicha facultad, los tomasinos, oradores e investigadores, fueron seleccionados mediante concurso público para participar y representar a la Santo Tomás.

Nuestra Institución ha participado en todas las ediciones de este concurso y en otros certámenes a nivel nacional, consolidando a nuestro claustro, y a la Facultad de Derecho, como uno de los principales centros de producción académica en materia de Derecho Penal Internacional en Colombia.

Como es usual, año tras año son múltiples los estudiantes que se preparan y se presentan para este concurso de gran relevancia internacional, donde participan algunas de las universidades de habla hispana más importantes; por 10 tanto, afrontar con solvencia la fase escrita y oral de este encuentro implica exigencia y alta calidad.

Este año la Santo Tomás ocupó el quinto lugar en la fase oral, por lo que celebramos esta exitosa participación producto del esfuerzo y la dedicación de nuestra docente y estudiantes, e invitamos a que todos aquellos interesados en participar en este valioso proyecto se inscriban en el semillero de Jurisprudencia Penal Internacional.

Noticia publicada en Tomás Noticias - 31 de julio de 2017

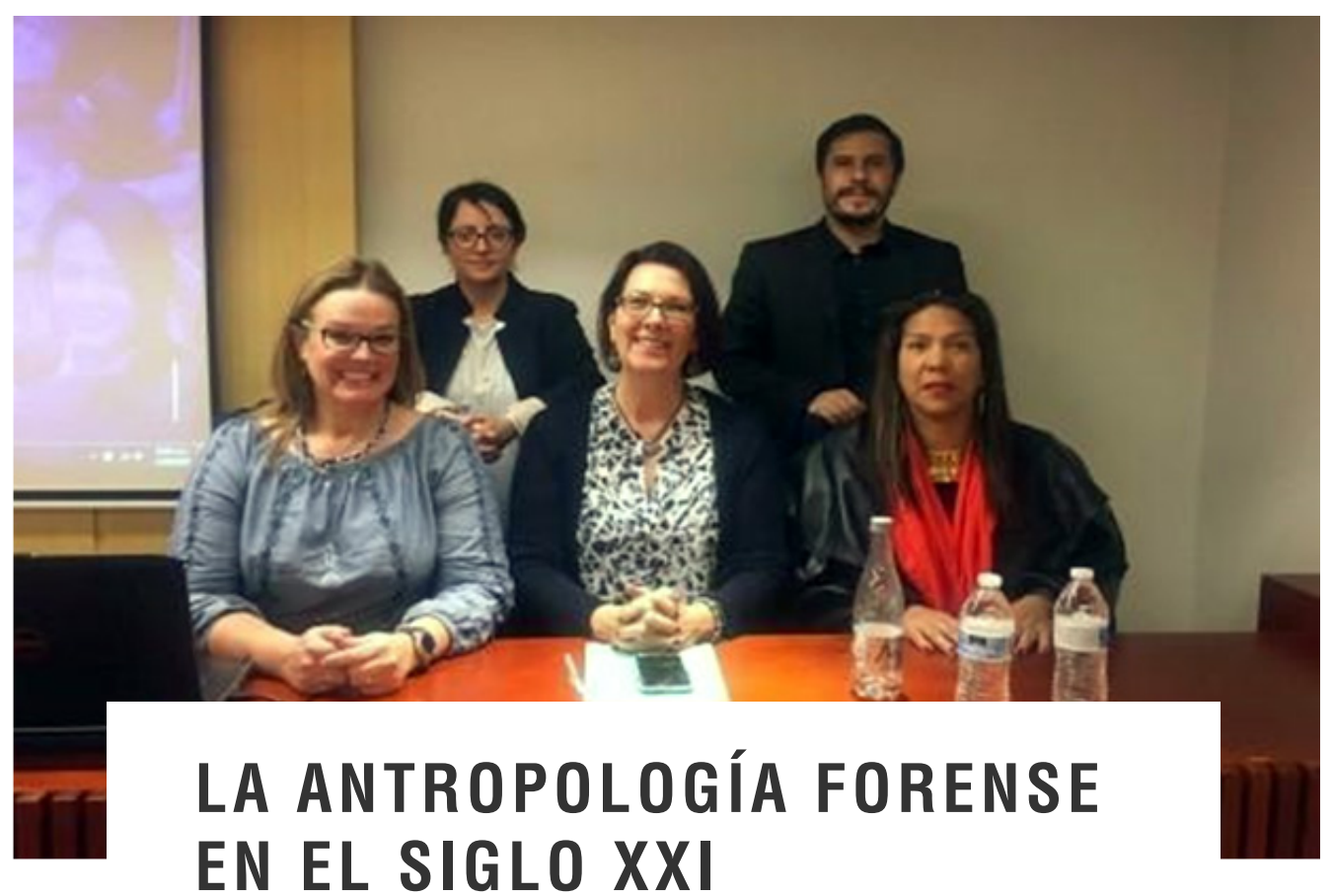

La Maestría de Derecho Penal y el módulo de Derecho Penal realizaron el conversatorio "La Antropología Forense en el siglo XXI, una conversación sobre las posibilidades y desafíos en casos reales".

Este conversatorio contó con la participación de la Dra. Heather Walsh Honey, Directora del Programa de Identidad Humana y Análisis de Trauma en el Departamento de Estudios de Florida Gulf Coast University; y la Dra. Victoria Sanford, maestra del Departamento de Antropología y Directora del Centro de Derechos Humanos y Estudios de Paz del Lehman College de la Universidad de Nueva York. 


\section{DOCENTE Y ESTUDIANTE DE LA UNIVERSIDAD CÉSAR VALLEJO DE PERÚ REALIZARON INTERCAMBIO ACADÉMICO EN LA SANTO TOMÁS}

El Dr. Luis Alberto León Reinaltt, Juez de Familia y docente de la Universidad César Vallejo de Perú, y la estudiante María Alejandra Guerra Gómez, de la misma institución, el pasado 2 de junio realizaron una estancia de carácter investigativo a la Facultad de Derecho de la Universidad Santo Tomás.

Con su visita se realizó un intercambio académico de los sistemas judiciales de Perú y de Colombia, concretamente en temas de oralidad en procesos por audiencias que incluyó el recorrido por algunas comisarías de familia y los tribunales Superior de Bogotá y Contencioso Administrativo de Cundinamarca.

La estudiante María Alejandra participó en diferentes clases con los estudiantes de nuestra Universidad, compartiendo con ellos el tema 'Asilo Político: caso Víctor Raúl Haya de la Torre'; mientras que el Dr. León Reinaltt realizó una presentación a la comunidad académica de la facultad sobre los temas: la estabilidad laboral, la violencia familiar y el proceso penal a menores de edad en el Sistema Judicial de Perú, permitiendo ver las diferencias y similitudes entre los sistemas legales manejados entre ese país y Colombia. Además, favoreció un espacio para el debate entre los estudiantes.

En la Comisaría de Familia de la localidad de Bosa realizaron una entrevista al Dr. Óscar Parra, Comisario de Familia, y Luz Mery Cárdenas, Trabajadora Social, sobre temas relacionados con la legislación de familia, la conciliación,

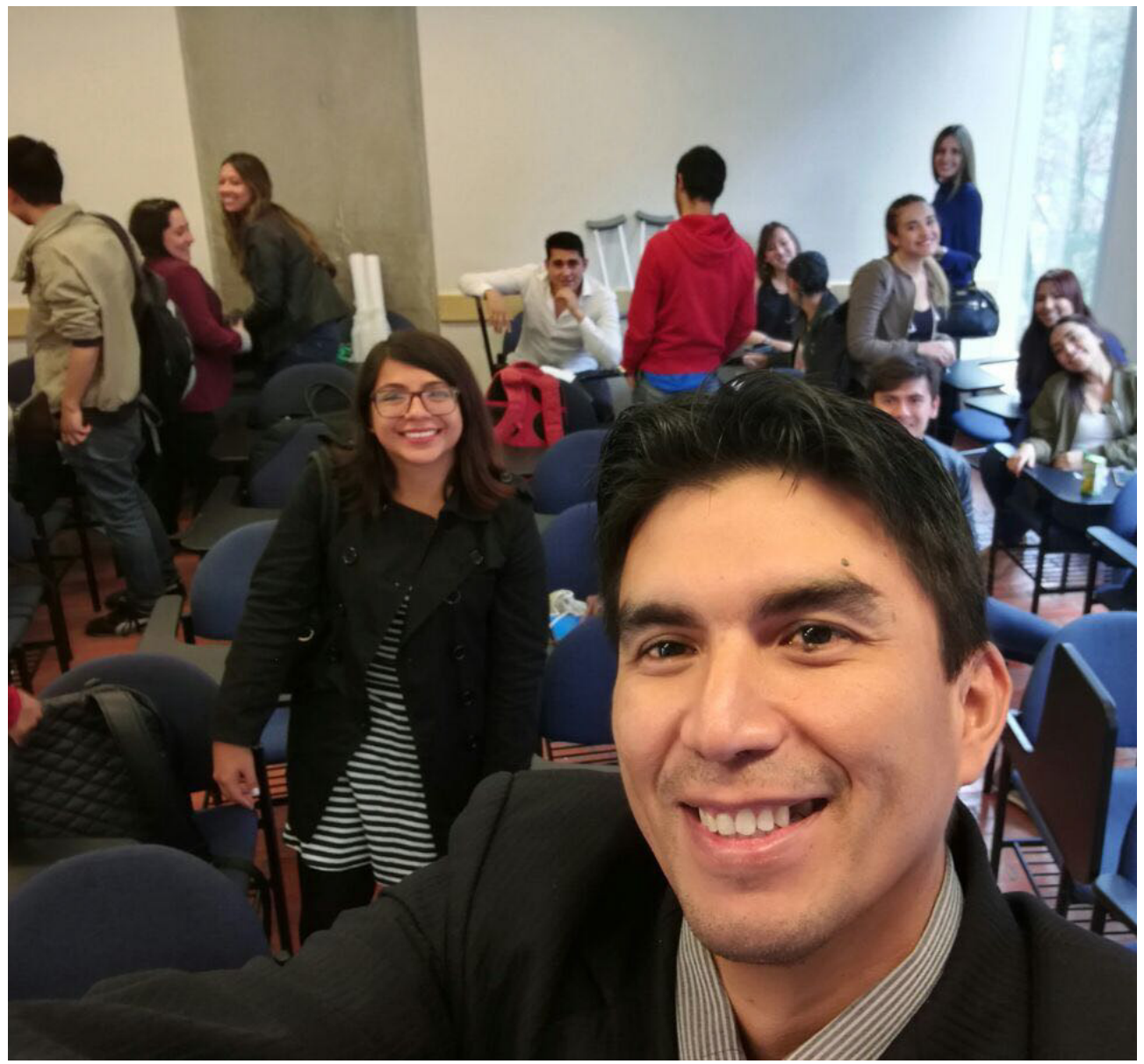

la cuota de alimentaria y las medidas de protección en el marco de la violencia intrafamiliar.

Entre tanto, a través de la asistencia a varias audiencias, la visita a las instalaciones de los tribunales Superior de Bogotá y Contencioso Administrativo de Cundinamarca favoreció el encuentro con magistrados para indagar sobre el procedimiento de oralidad.

Durante la actividad realizaron una visita guiada con el profesor Javier Rocha Amaris, docente de la Facultad de Derecho de la Universidad Santo Tomás y Exjuez Administrativo del Circuito a los Despachos del Tribunal Administrativo de Cundinamarca.

Esta enriquecedora experiencia ha dejado las puertas abiertas a los tomasinos para realizar próximos encuentros con la comunidad académica de la Universidad César Vallejo de Perú, quienes han manifestado estar complacidos de recibir a nuestros estudiantes en sus instalaciones en la ciudad de Trujillo.

Noticia publicada en Tomás Noticias - 31 de julio de 2017 


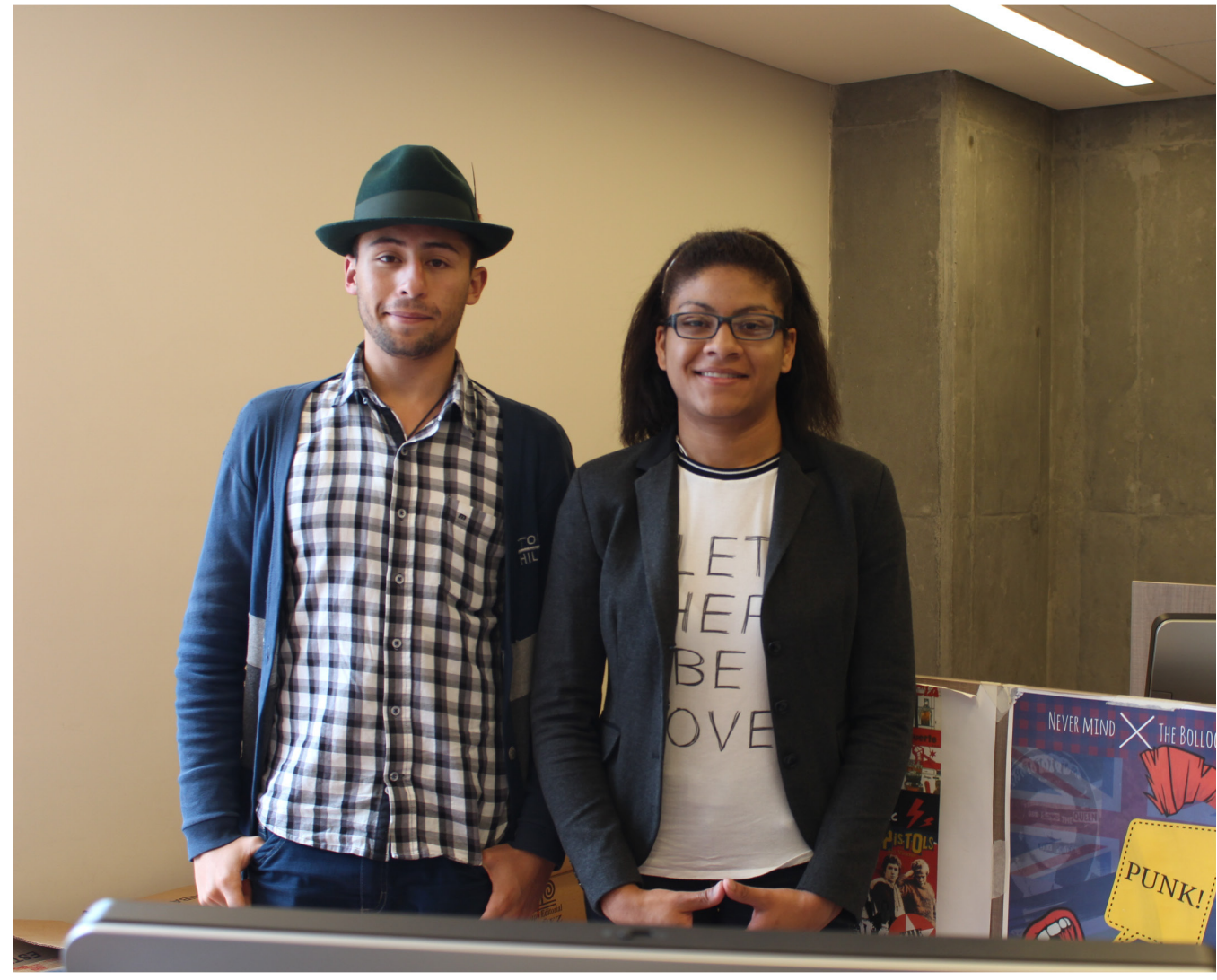

INVESTIGACIÓN DE USTA COLOMBIA PRESENTE EN EL X ENCUENTRO NODO CENTRO DE LA RED DE GRUPOS Y CENTROS DE INVESTIGACIÓN JURÍDICA Y SOCIOJURÍDICA

La Universidad Santo Tomás, a través de la Facultad de Derecho, participó en el $X$ Encuentro Nodo Centro de la Red de Grupos y Centros de Investigación Jurídica y Sociojurídica, que se realizó en el primer semestre del año en curso en la Universidad La Gran Colombia.

La intervención de nuestra Universidad inició con las ponencias de los estudiantes Daniela Valencia y Esteban José Ahumada, del Semillero de Investigación Hermenéutica y Argumentación Jurídica, liderado por la Doctora Dalia Carreño.
Entre los temas que representaron a la Universidad Santo Tomás Sede Bogotá se destacan las ponencias: 'Marco jurídico para la inteligencia artificial aplicada a los robots como sistemas autónomos', de Daniela Valencia y 'Manipulación genética y su influencia en el sistema legal colombiano como reconfiguración del derecho a la salud', de Esteban José Ahumada, ambas construidas en el marco del semillero.

Por otro lado, la Sede Villavicencio participó con la ponencia 'El ciber-control parental: un mecanismo idóneo para salvaguardar el interés superior del menor, frente a los riesgos online', del semillero Derecho Informático y de las Tecnologías en la Sociedad de la Información (DITSI), cuyos ponentes fueron Cristian Anderson Rodríguez Basallo, Sebastián Caicedo Cerón y Laura Arias.

Así mismo, se contó con la ponencia 'La decadencia juvenil en Colombia como reto del post-acuerdo', del semillero de investigación Teoría del Estado y la Constitución, expuesta por Jessica Paola Espitia Parra y Germán Andrés Martínez Mateos, de la Seccional Tunja.

De esta manera se concluyó una magnifica jornada con pares académicos de varias universidades que acompañaron a los estudiantes en sus presentaciones para así mejorar cada vez más la producción académica investigativa, contribuir en la formación de cada participante y fortalecer su capacidad investigativa.

Noticia publicada en Tomás Noticias - 28 de agosto de 2017 


\section{PUBLIGAGIONES}

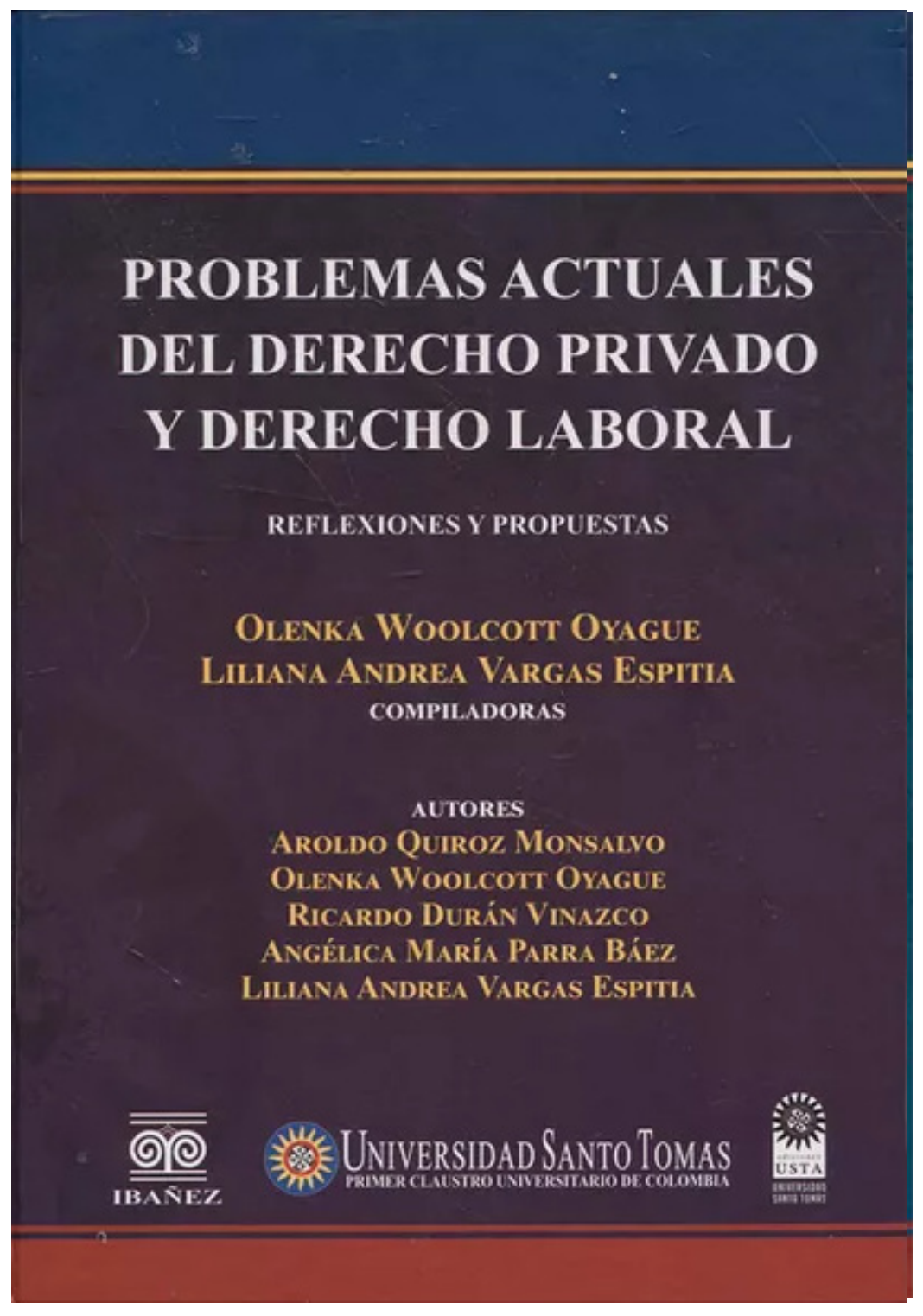

\section{NOVEDADES}

- Tendencias Jurídicas del Derecho Público por Carlina Blanco Alvarado.

- Bioética y Docencia por Dalia Carreño Dueñas y otros.

- Iusfilosofía con ventanas por Juan Guillermo Durán.

- Historiografía persona y nación por Dalia Carreño Dueñas y David Valencia.

- Problemas Actuales del Derecho Privado y Derecho Laboral por Olenka Woolcott y otros.

- Libertad de los jueces para fallar en positivo por Édgar Guarín.

- Derecho Penal Vigilancia y Control Social por Juan Francisco Mendoza y otros.

- Oralidad y Derecho por Álvaro Moreno Durán y otros.

- Las tendencias entre libertad y seguridad por Ciro Güechá Medina y otros.

\section{REVISTA EMPEIRÍA}

Esta revista, escrita para estudiantes por los estudiantes, presenta los resultados de las investigaciones con el fin de enseñar a la comunidad la producción académica y los avances de los estudiantes que están realizando a través del departamento.

Siendo esta una revista de los estudiantes de la Universidad Santo Tomás, con miras a incentivar la producción académica por medio de la investigación, se invita a nuestra creciente comunidad de estudiantes de Derecho a que participen en estas producciones.

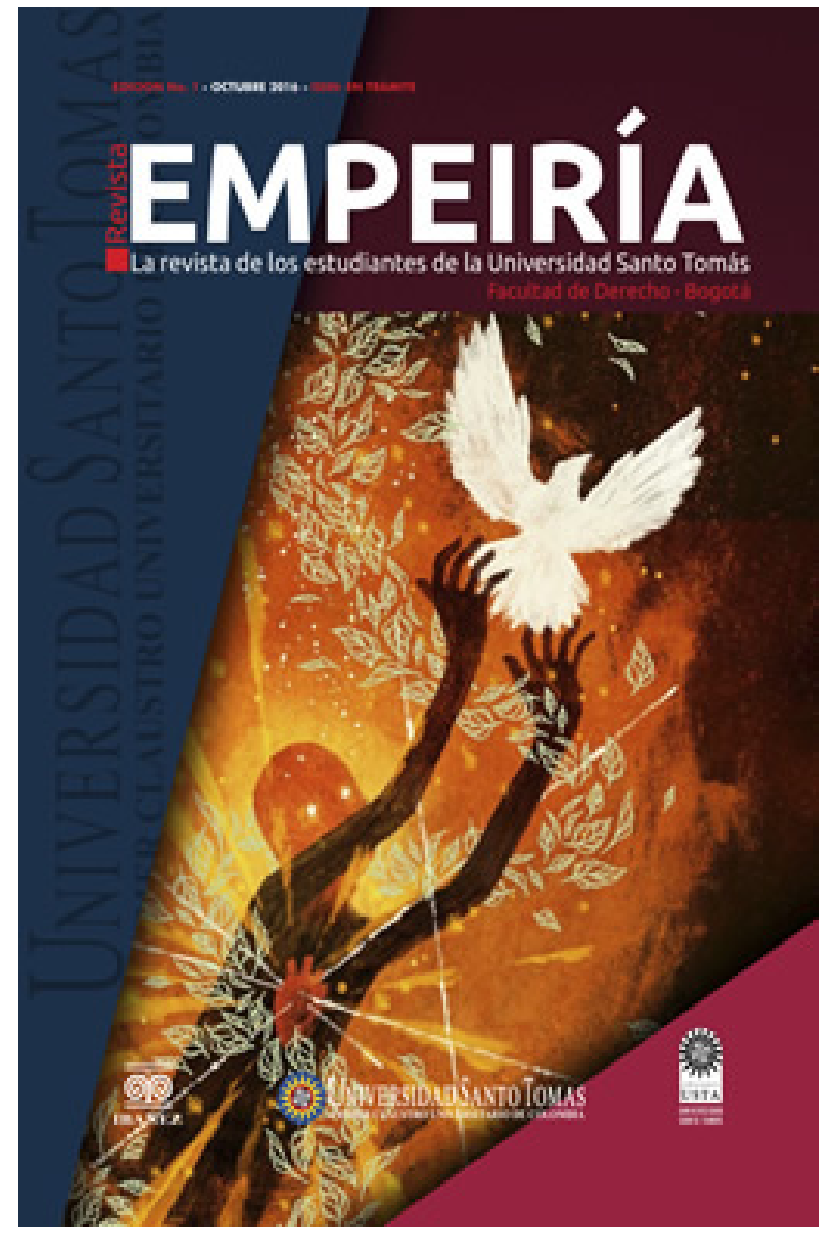




\section{NUESTRAS MAESTRIAS}
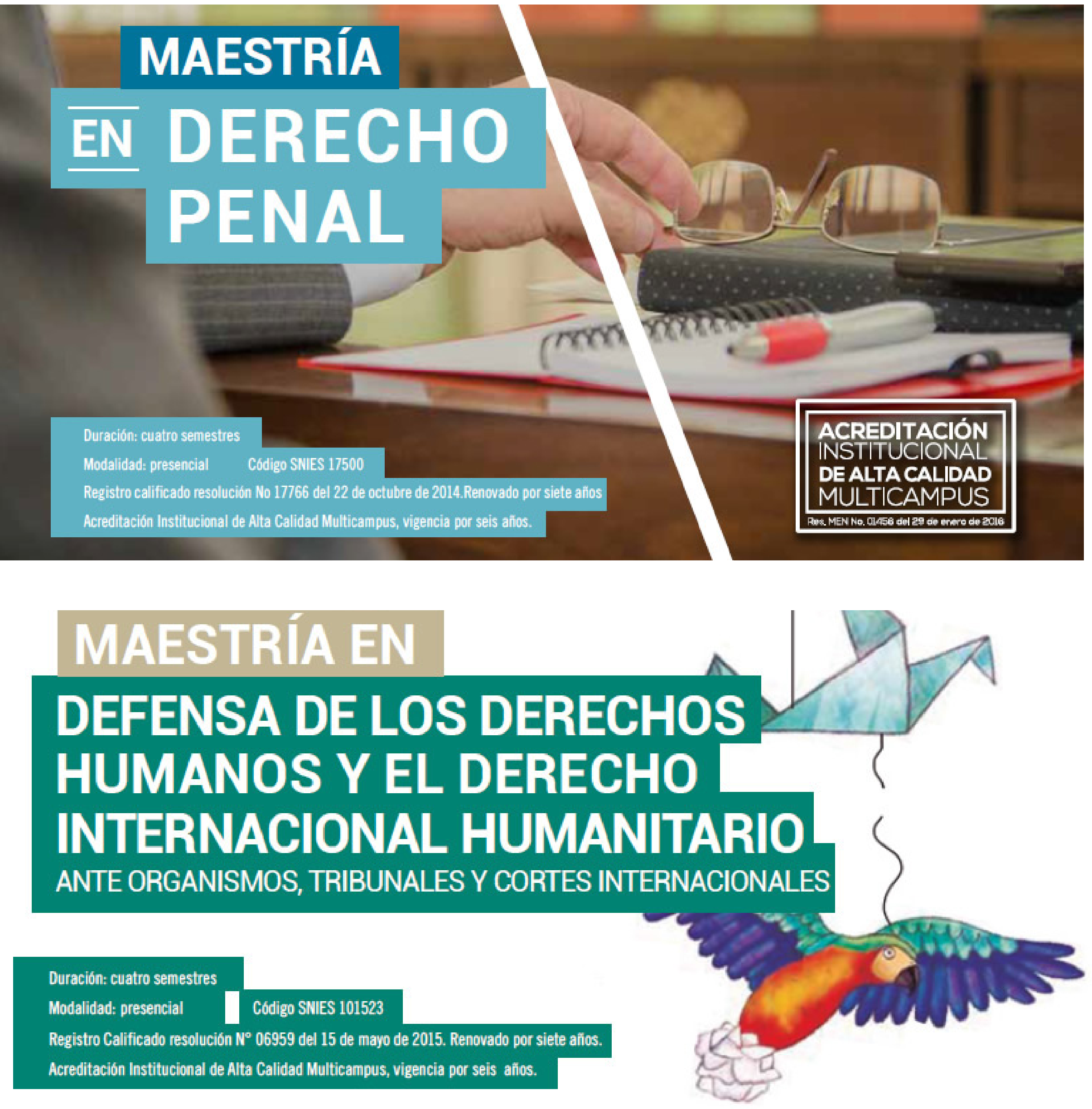

Nuestro Claustro ofrece múltiples maestrías que tienen por objeto la profundización en materias específicas ampliando el conocimiento de los estudiantes y sus habilidades, entre ellas encontramos la Maestría de Derecho Penal, Ia Maestría de Derecho Público, la Maestría en la Defensa de Ios Derechos Humanos y del DIH ante organismos, Tribunales y Cortes Internacionales, y la Maestría en Derecho Contractual Público y Privado. 


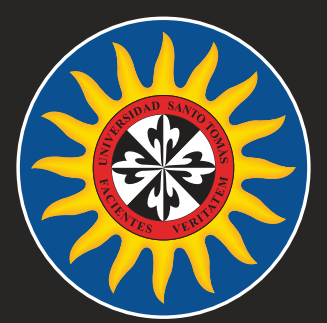

\section{UnIVERSIDAD SANTOTOMAS PRIMER CLAUSTRO UNIVERSITARIO DE COLOMBIA}

